\title{
Editorial
}

\section{Verification Approaches for Nano- and Micro-Satellites}

\author{
Dario Modenini * (D) and Paolo Tortora * (D) \\ Dipartimento di Ingegneria Industriale, Alma Mater Studiorum Università di Bologna, 47121 Forlì, Italy \\ * Correspondence: dario.modenini@unibo.it (D.M.); paolo.tortora@unibo.it (P.T.); \\ Tel.: +39-0543-374450 (D.M.); +39-0543-374456 (P.T.)
}

Received: 7 April 2020; Accepted: 7 April 2020; Published: 8 April 2020

\section{Introduction and Scope}

There is growing interest for the development of light, small, high-performance spacecraft (S/C) platforms for a wide range of missions. In the early stages of the small-satellite era, both nano-satellites $(<10 \mathrm{~kg})$ and micro-satellites $(>10 \mathrm{~kg})$ were mainly intended for educational and technology demonstration goals [1]. Nowadays, they are a consolidate means for Earth observation, where they dramatically reduce mission costs. We are now at a turning point, where nano- and micro-satellite systems can accomplish interplanetary missions beyond the boundaries of low Earth orbit (LEO) $[2,3]$. However, in spite of the substantial increase in low-mass satellites launched since 2013, several statistics show the low success rate of these commercial off-the-shelf (COTS)-based cost-driven systems. Only half of nano-satellites succeeded in mission operations after a successful launch in the last 15 years [4].

The low success rate of nano-satellites is acceptable, up to a certain extent for educational or technology demonstration missions. This may stem from the way in which university-led projects design and carry out the $\mathrm{S} / \mathrm{C}$ ground verification process, which lacks repeatability and rigor routinely found in industry. However, even though a nano-satellite is relatively inexpensive, if the S/C is launched for commercial or scientific exploration purposes, failure is not really an option.

We should thus ask ourselves what technical challenges and programmatic difficulties must be faced in order to substantially increase the reliability of nano- and micro-satellite missions. The historical causes of low-mass satellite failure can be traced back to (a) lack of system-level testing due to schedule and budget constraints, (b) inadequate thermal design and verification, and (c) use of COTS electronics. Clearly, rigorous ground verification approaches - tailoring the existing testing standards for traditional large-/medium-class satellites-are needed to effectively face such challenges.

It is therefore our pleasure to introduce this Aerospace MDPI Special Issue on Verification Approaches for Nano- and Micro-Satellites, which aims at addressing, at least in part, the above concerns.

\section{Contributions}

Twelve high-quality papers were submitted to this Special Issue, covering several topics ranging from surveys of past experiences, novel approaches towards reliability analysis and Assembly, Integration, and Verification (AIV) assessment, testing facilities, to in-flight experience and on-orbit anomaly investigation.

As recalled in Section 1, nano-satellites are an invaluable educational tool for universities. Alanazi and Straub [5] provide a survey of student-driven CubeSat projects designed by students and faculty members. They present data to identify the challenges and needs of CubeSat developers, investigating reasons for a mission failure from students' perspectives. Monteiro et al. [6] report on the AIV approach they developed for the ISTSat-1 educational satellite, showing how testing functional prototypes at an early development stage led to uncovering system-level errors. The insights gained 
from the early tests described in this work would have been more difficult, if not impossible, to gain if employing a traditional "stage-gate" approach, under which integration testing would take place only after each individual subsystem is verified.

The issue of low reliability in nano- and micro-satellites is directly faced in the work by Menchinelli et al. [7], who push forward a reliability engineering approach based on Failure Mode, Effects, and Criticality Analysis (FMECA) to manage CubeSat reliability data and prioritize criticalities early in the design phase. Applying a risk analysis methodology during the design phase offers the opportunity to implement corrective actions at little to no cost. Their approach was applied during the design phase of a 6-Unit university CubeSat, allowing for identification of the major criticalities of the design.

Five research papers in this Special Issue are devoted to verification strategies for some mission-critical subsystems, such as the Attitude Determination and Control Subsystem (ADCS), the Thermal Control Subsystem (TCS), and the Power System (PS). Kiesbye et al. [8] report the development of a framework for hardware and software-in-the-loop verification of MOVE-II (Munich Orbital Verification Experiment 2) CubeSat, with a strong focus on the ADCS, showing how we can enhance the reliability of CubeSats through system-level testing. Farissi et al. [9] present and experimentally verify an attitude control strategy based on magnetometer-only measurements for detumbling and pointing of nano-satellites, to be used as a backup solution in case of failure of the primary ADCS, and preventing mission loss. Modenini et al. [10] report the in-lab development of a dynamic testbed for nano-satellite attitude verification, placing focus on the thorough performance assessment of all the subsystems building up the facility. Piedra, Torres, and Ledesma [11] explore the viability, from a thermal point of view, of a composite material with $\mathrm{ZnO}$ nanoparticles to be employed in lieu of aluminum for the primary structure of a CubeSat, by performing finite element thermal analysis. After recognizing scalability as an effective approach towards rapid subsystems development times, while reducing the cost and failure rate, Gonzalez-Llorente et al. [12] present a modular approach for the power generation within micro-satellites, called the Solar Module Integrated Converter (SMIC), using the Ten-Koh micro-satellite as a testbed to prove this concept.

Miniaturized electric propulsion may dramatically increase the range of missions achievable with nano-satellites. However, relatively low readiness level and unease to perform thorough tests are slowing down the spread of electric propulsion solutions. The work of Stesina [13] provides a flexible test platform to assess the mutual interaction between the electric propulsion systems with the hosting CubeSat platform interactions in terms of power consumption, chemical contamination, and generated thermal and electromagnetic environments from mechanical, electrical, magnetic, and chemical perspectives.

Beyond the space segment, the launch segment has also been considered by the contributors of this Special Issue. Indeed, nano and micro launch vehicles (NMLVs) may become a viable solution to make launch costs of nano- and micro-satellites more competitive. Eramo et al. [14] present an experimental investigation of an Ethernet-based communication system to be implemented onboard NMLVs for interconnecting with the hosted payloads, showing how their solution has performance comparable to the much more expensive alternative, the TTEthernet standard.

Finally, valuable in-flight experiences are reported in works by Kim, Nam, and Jung [15] and Stesina and Corpino [16], demonstrating the importance of ground simulation facilities aimed at subsystems performance verification and investigation of in-orbit anomalies, respectively.

\section{Conclusions and Outlook}

More than one year after the initial call for contributions, we may certainly say this Special Issue has been a success: almost all the intended research areas mentioned in the call have been covered by the Authors, and valuable lessons learnt have been reported. Most notably, the manuscripts provide strong evidence on the importance of an early approach to system integration, possibly starting even before subsystems completion. Such an approach may help in identifying system-level design flaws at 
a point when they can be recovered with minimal impact on the overall project schedule, which is of paramount importance for those programs leveraging on rapid deployment times, as typical for nanoand micro-satellite missions.

Dario Modenini and Paolo Tortora wish to thank all the contributors to this Special Issue, including the Authors, Reviewers, and MDPI Editors.

Conflicts of Interest: The authors declare no conflict of interest.

\section{References}

1. Swartwout, M. Reliving 24 Years in the Next 12 Minutes: A Statistical and Personal History of University-Class Satellites. In Proceedings of the 32nd Annual AIAA/USU Conference on Small Satellites (SSC18-WKVIII-03), Logan, UT, USA, 4-9 August 2018.

2. Schoolcraft, J.; Klesh, A.; Werne, T. MarCO: Interplanetary Mission Development on a CubeSat Scale. In Proceedings of the 14th International Conference on Space Operations, Daejeon, Korea, 16-20 May 2016.

3. Walker, R.; Binns, D.; Bramanti, C.; Casasco, M.; Concari, P.; Izzo, D.; Feili, D.; Fernandez, P.; Fernandez, J.G.; Hager, P.; et al. Deep-space cubesats: Thinking inside the box. Astron. Geophys. 2018, 59, 5-24. [CrossRef]

4. Koudelka, O. Micro/Nano/Picosatellite-activities: Challenges towards space education and utilization. In Small Satellites; Marboe, I., Ed.; Brill|Nijhoff: Leiden, The Netherlands, 2016; pp. 11-13.

5. Alanazi, A.; Straub, J. Engineering Methodology for Student-Driven CubeSats. Aerospace 2019, 6, 54. [CrossRef]

6. Monteiro, J.P.; Rocha, R.M.; Silva, A.; Afonso, R.; Ramos, N. Integration and Verification Approach of ISTSat-1 CubeSat. Aerospace 2019, 6, 131. [CrossRef]

7. Menchinelli, A.; Ingiosi, F.; Pamphili, L.; Marzioli, P.; Patriarca, R.; Costantino, F.; Piergentili, F. A Reliability Engineering Approach for Managing Risks in CubeSats. Aerospace 2018, 5, 121. [CrossRef]

8. Kiesbye, J.; Messmann, D.; Preisinger, M.; Reina, G.; Nagy, D.; Schummer, F.; Mostad, M.; Kale, T.; Langer, M. Hardware-In-The-Loop and Software-In-The-Loop Testing of the MOVE-II CubeSat. Aerospace 2019, 6, 130. [CrossRef]

9. Farissi, M.S.; Carletta, S.; Nascetti, A.; Teofilatto, P. Implementation and Hardware-In-The-Loop Simulation of a Magnetic Detumbling and Pointing Control Based on Three-Axis Magnetometer Data. Aerospace 2019, 6, 133. [CrossRef]

10. Modenini, D.; Bahu, A.; Curzi, G.; Togni, A. A Dynamic Testbed for Nanosatellites Attitude Verification. Aerospace 2020, 7, 31. [CrossRef]

11. Piedra, S.; Torres, M.; Ledesma, S. Thermal Numerical Analysis of the Primary Composite Structure of a CubeSat. Aerospace 2019, 6, 97. [CrossRef]

12. Gonzalez-Llorente, J.; Lidtke, A.A.; Hatanaka, K.; Kawauchi, R.; Okuyama, K.-I. Solar Module Integrated Converters as Power Generator in Small Spacecrafts: Design and Verification Approach. Aerospace 2019, 6, 61. [CrossRef]

13. Stesina, F. Validation of a Test Platform to Qualify Miniaturized Electric Propulsion Systems. Aerospace 2019, 6, 99. [CrossRef]

14. Eramo, V.; Lavacca, F.G.; Valente, F.; Pisculli, A.; Caporossi, S. Simulation and Experimental Evaluation of a Flexible Time Triggered Ethernet Architecture Applied in Satellite Nano/Micro Launchers. Aerospace 2018, 5, 84. [CrossRef]

15. Kim, S.; Nam, T.; Jung, D. Experimental Validation of an Onboard Transient Luminous Events Observation System for VisionCube via Ground Simulation Environment. Aerospace 2018, 5, 100. [CrossRef]

16. Stesina, F; Corpino, S. Investigation of a CubeSat in Orbit Anomaly through Verification on Ground. Aerospace 2020, 7, 38. [CrossRef]

(C) 2020 by the authors. Licensee MDPI, Basel, Switzerland. This article is an open access article distributed under the terms and conditions of the Creative Commons Attribution (CC BY) license (http://creativecommons.org/licenses/by/4.0/). 